\title{
Experience with Blood Brain Barrier Disruption in the Treatment of Malignant Brain Tumor
}

\begin{abstract}
J. Devkota, M.D.
DOI: http://dx.doi.org/10.5915/17-2_3-12753

Abstract:

The blood-brain-barrier (BBB) seems to be a major factor in the paucity of chemotherapeutic agents entering the brain to be effective against the malignant brain tumor. Since systemic treatment with large doses of chemotherapeutic agents provide no benefit, a transient reversible osmotic opening of this barrier is necessary. Intraarterial infusion of hyperosmolar mannitol into the cerebral tumor area disrupts the BBB and thus immediate intra-arterial infusion of multiple drugs enabled them to reach the tumor in a much higher concentration. This disruptive mechanism and mode of delivery of drug appeared to be the only currently available effective and dependable pathway.
\end{abstract}

\section{Introduction}

Treatment of recurrent brain tumor has been a great concern over the years. In spite of effective chemotherapy for a variety of other tumors of the body, brain tumor remains isolated, unapproachable and untouchable by conventional methods of chemotherapy. This is due to the presence of the blood-brain barrier (BBB) which prevents a majority of substances from crossing it; thus the ineffectiveness of the oral and intravascular routes of chemotherapy for brain tumors. It has been proven that the brain blood capillary endothelium and basement membrane have tight junction between the cells (zona occludes), nonfenestration, paucity of vascular transport (pinocytosis) and closely investing glial sheath composed of the "end feet" of astrocytes.' One alternative to the delivery of a large dose of chemotherapeutic agent across this impermeable blood-brain-barrier (BBB) is to disrupt it with hyperosmolar agent (mannitol) given through cerebral intra-arterial injection. The aim is to completely replace the blood supply of the tumor by mannitol for a period of 25 to 35 seconds. The total volume and rate of mannitol injection is critical to produce proper disruption without inducing hypertensive encephalopathy or sub-disruption. The chemotherapeutic agent methotrexate (MXT) given intraarterially and cytoxan (CYTX) given intravenously can traverse the transiently disrupted BBB to reach the tumor area. These two substances are found to be the least neurotoxic. Other therapeutic agents are also available but they are very neurotoxic.

Reversible transient BBB disruption was achieved in six patients who were proven to be incurable after

From the Department of Radiology, University of Missouri-Columbia, Health Sciences Center, Columbia, Missouri 65212

Address all correspondence to Dr. J. Devkota, Assistant Professor, Chief, Neuroradiology, Department of Radiology, University of MissouriColumbia Health Sciences Center, Columbia, Missouri 65212 surgery, complete radiation and chemotherapy. Their life expectancy was very short. Following BBB disruption and intra-arterial chemotherapy, their quality of life improved remarkably and they remained relatively independent of medications. Each patient was monitored by computerized brain tomography (CT) It was found that the first BBB disruption procedure provided a dramatic reduction of the mass effect as a result of a decrease in surrounding edema. The tumor itself showed a small response. The patients improved substantially, felt better and gained weight, although there was no restoration of neurological loss.

\section{Materials and Methods}

Eighteen BBB disruption procedures on 6 patients were performed. Four patients were young (27-33 years) with primary malignant astrocytoma. The fifth patient, aged 62 years, had glioblastoma multiformis. Only the sixth patient had a posterior fossa metastasis from malignant prostatic carcinoma. Incidentally, 3 of the malignant astrocytoma were on the right side. These patients had surgery once or twice followed by a full course of radiation (6000R) and chemotherapy. There was no decrease in tumor size or activity by this treatment. The patients had very poor health and nausea with signs of elevated intracranial pressure. They had variable degrees of neurological deficit. Their life expectancy was counted in days. The only last resort was to perform a BBB disruption procedure.

Informed consent was obtained from the patient and the family. Complete clinical and laboratory tests were performed for the suitability of the patient for the BBB disruption procedure.

A basal pretreatment computed tomography (CT) of the head was obtained. Under light general anaesthesia the patient was placed in a supine position and utilizing the Sildenger technique, a femoral puncture was made. A 6.5 French cerebral catheter $\left(\mathrm{H}_{1}\right)$ was advanced into the internal carotid artery and a complete set of frontal and lateral view carotid ( 5 cases) and vertebral arteriograms (one case) was obtained to evaluate the status of the carotid supply, tumor vas- 
cular status and the Circle of Willis. In subsequent BBB disruption procedure no arteriogram was obtained but each time CT of the head was utilized as a guide.

Repeat trial of a test dose injection of radio-opaque medium (conray) through the carotid arterial catheter by an automatic pressure injector ( 450 pound per square inch) was made to reflux into the external carotid artery and an average of $8 \mathrm{ml} / \mathrm{sec}$. was sufficient for this purpose. For the vertebral artery, reflux into the contralateral vertebral artery was observed with $6.5 \mathrm{ml} / \mathrm{sec}$. Mannitol $25 \%(250 \mathrm{ml})$ was infused through the intra-arterial catheter after determining the total volume and rate from the refluxing test dose. Immediately after mannitol infusion, methotrexate (MTX) $1500 \mathrm{mg}$ was administered through the same intra-arterial catheter. Cytoxan (20-40 mg) was given intravenously about 10 minutes prior to the mannitol infusion. The radioisotope $99 \mathrm{mT}$ c DTPA, $30 \mathrm{mCi}$ was also given intravenously just before cytoxan. After completing drug administration, the patient was sent to the neurosurgical intensive care unit. Three hours later the patient was brought to the nuclear medicine section for a brain scan.

\section{Neuroradiology Technical Observation:}

As a clinical trial, a 6.5 French head hunter $\left(\mathrm{H}_{1}\right)$ catheter and long floppy (Bentson) guide wire in the case of younger patients ( 5 cases) and $\mathrm{HN}_{4} 5$ French with 32 floppy wire in older patients were chosen. It was found that $\mathrm{H}_{1}$ catheter (6.5) gave a good torque to adjust the catheter into the internal carotid artery. A good reflux with $7 \mathrm{ml} / \mathrm{sec}$ with total volume of $20 \mathrm{ml}$ was obtained. In a subsequent BBB disruption procedure on the same patient, a higher rate of flow of the test dose was required and variable degrees of spasm was also seen. Even a slight accentric position of the catheter tip triggered transient spasm which was obviated by slowing the infusion rate of physiological saline solution or if further prolonged spasm was encountered, $1 \mathrm{ml}$ of lidocaine in $5 \mathrm{ml}$ saline was infused through the intra-arterial catheter. Although the total volume and rate of contrast agent could be delivered satisfactorily through the $5 \mathrm{~F}$ catheter, a $6.5 \mathrm{~F}$ catheter was found to be more suitable. Adequate reflux and less need for manipulation of the catheter were advantages of the $6.5 \mathrm{~F}$ catheter. The transient but recurrent spasms of the internal carotid artery was annoying. As a result the fluoroscopy and catheter time was long. Distal the position of the catheter tip into the internal carotid artery greater was the degree of spasm in the cerebral arteries. Also the irritability of the internal carotid artery was proportional to the brain tumor activity. The least possible manipulation of the guide wire and cerebral catheter was found to be an essential aspect of the procedure. The effective blood replacement in cerebral vasculature by the mannitol was monitored by observing the ipsilateral blanching of the face and conjunctiva as well as medriasis in the ipsilateral eye. Blanching of those areas suggests complete wash out of the blood by mannitol to ensure BBB disruption. This phenomenon lasted as long as the mannitol injection was made. This interesting phenomenon of occular medriasis was observed in 1 patient even during the injection of the test dose of contrast (conray - 60). With each injection the medriasis became more and more persistent so the procedure had to be stopped at that point to be rescheduled on a later date.

\section{Results}

Of the six patients all of them had BBB disruption procedures performed twice or more. One young patient showed another focus in the posterior fossa in addition to the primary lesion in the right hemisphere after first BBB disruption procedure which cleared after third BBB disruption. These patients had completed radiation and chemotherapy, were feeble, hemiparetic, unbalanced, nauseated, poorly fed and hopeless. Their life expectancy was extremely short. Following first BBB disruption procedure, all patients with primary astrocytoma showed remarkable improvement from physical and emotional standpoint. They gained weight and strength. Thus, their life style changed completely for the better. A followup CT scan of the head showed reduction in the mass effect and brain edema with some reduction in the size of the main tumor bulk. These findings encouraged further treatment. With each successive treatment, the degree of response by the tumor was less but the growth of the tumor and the surrounding edema continued to be restricted. There appeared to be a plateau of response after the third or fourth course of treatment. These patients were monitored by contrast enhanced CT scan of the head. Although the CT finding was not dramatic, the physical and emotional status of the patients were gratifying. They were less dependent on medications and their life expectancy increased. The effective BBB disruption and its degree was accessed by three hours post BBB disruption isotope scan of the brain which showed diffuse increase uptake of the isotope in the tumor area which was not present in the pre-BBB disruption procedure scan. Because of time, technical difficulties, and convulsive response of the patients, no immediate post BBB disruption CT scan was obtained.

None of the patients showed any evidence of further metastasis of the lesion to other areas.

\section{Discussion}

It is evident that the BBB prevents passage of molecules in the systemic circulation to the brain. Thus, there is no benefit from systemic chemotherapy of brain tumors even when given in large doses. The only current way to deliver an effective intracranial dose 
of chemotherapy is through intra-arterial cerebral infusion immediately after BBB disruption. From our small series and early experience, it is clear that apparently incurable and miserable patients can be improved and expect a better quality of life.

The concept of BBB is explained in terms of neuronal capillary endothelial wall impermeability to many molecules commonly present in the systemic circulation or injected from outside. 1

Not all substances are impermeable to the BBB. High lipid solubility, ${ }^{2}$ low degree of ionization at physiological $\mathrm{Ph}$ and lack of plasma protein binding permit ready passage across the BBB and rapid equalization. ${ }^{3,4}$ Being a near perfect semipermeable membrane, the BBB allows water to move in either direction to maintain equal osmotic concentration of solutes in cerebral extracellular fluid and blood.1.5 Additionally, solutes such as potassium $(\mathrm{K})$ calcium (Ca) and magnesium (Mg) and water are maintained in a very constant cerebrospinal fluid concentration despite severe and prolonged disturbances in blood plasma concentrations. 2,5

Certain chemotherapeutic agents, such as cisplatinum, cross the BBB with their own disruptive capacity but Bleomycin and 5-fluoro-uracil do not cross without BBB disruption. These drugs are found to be effective but highly neurotoxic, so their use is limited. Cytoxan was found to be the least neurotoxic. It is given intravenously just prior to BBB disruption because it requires metabolic transformation by the liver. ${ }^{6}$ Methotrexate is widely known and used for treatment of several neoplasms with effective results. However, to be effective against brain tumors, it needs to cross the BBB and therefore has to be given through intra-arterial infusion following BBB disruption. All our patients received MTX immediately following osmotic BBB disruption. The level of MTX was found to be 10 to 100 -fold higher in the brain with this delivery system. ${ }^{7}$ The effective half-life of MTX is 30 to 90 minutes. MTX is cell division phase specific and most effective in the S-phase of DNA synthesis in the cell cycle. The cell division cycle of an astrocytoma ranges from 2 to 50 days, MTX has to be present for at least 2 days within the tumor area to be effective.$^{8}$ Fortunately, the effective concentration of MTX is maintained within the tumor for this period of time or longer because MTX does not cross the BBB and diffuse outside the brain. Clinical studies have demonstrated that $\mathrm{BBB}$ can be reversibly disrupted in both the cerebrum and the posterior fossa without significant toxicity $11-13$ and that the degree, location and duration of barrier modification can be monitored by CT or radionuclide brain scan. Our experience along with that of others ${ }^{12}$ indicate that multiagent (cytoxan and MTX) chemotherapy carries no significant neurotoxicity or permanent complications. The MTX was chosen in our study because of its low toxicity on direct exposure to brain tissue, reported responses of brain tumors to this agent and the possibility of a reliable assay method.

In conclusion, chemotherapeutic agents or antibodies must cross the BBB to be effective. Instead of trying systemic chemotherapy, intra-arterial injection following BBB disruption, appears to be the only current way to deliver a tissue therapeutic dose either for the treatment of intracranial cancers or infections. Hence, this procedure is not only indicated for cancer patients but possibly also for many other central nervous system disorders.

\section{References}

1. Sage M: Blood-brain barrier-phenomenon of increasing importance of the imaging clinicians. AJR 138:887-898, 1982.

2. Katzman R, Pappius HM: Brain electrolytes and fluid metabolism. Baltimore, Williams \& Wilkins, 1973.

3. Mayer SE, Maickel RP, et al: Kinetics of penetration of drugs and other foreign compounds into the cerebrospinal fluid and brain. J. Pharmacol 127:205-211, 1959.

4. Brodie BB, Kurz $\mathrm{H}$, et al: The importance of dissociation constant and lipid solubility in influencing the passage of drugs into cerebrospinal fluid. J Pharmacol 130:20-25, 1960.

5. Bradbury M: The concept of blood brain barrier. New York, J. Wiley, 1979.

6. Neuwelt EA, Burnett PA, et al: Pharmacology and neurotoxicity of cis-diamminedichloroplatinum, bleomycin, 5-fluorouracil and cyclophosphamide administration following osmotic BBB modification. Cancer Research 43:5278-85, 1983.

7. Neuwelt EA, Glasberg M, et al: Neurotoxicity of chemotherapy agent after blood-brain-barrier modification. Neuropathological studies. Ann Neurol 14:316-324, 1983.

8. Neuwelt EA, Frankel EP, et al: Effect of osmotic blood brain barrier disruption on methotrexate pharmacokinetics in the dog. Neurosurgery 7:36-43, 1980.

9. Neuwelt EA, Balahan E, et al: Successful treatment of primary central nervous system lymphoma with chemotherapy after osmotic blood-brain-barrier opening. Neurosurgery 12:662-671, 1983.

10. Neuwelt EA, Diehl JT, et al: Monitoring of methotrexate delivery in patients with malignant brain tumors after osmotic blood-brain-barrier disruption. Ann Intern Med 94:449-454, 1981.

11. Neuwelt EA, Frenkel EP, et al: Reversible osmotic bloodbrain-barrier disruption in humans: Implications for the chemotherapy of malignant brain tumors. Neurosurgery 7:44-52, 1980.

12. Neuwelt EA, Diehl JT, et al: Ann Intern Med 194 (Part 1): $449-454,1981$. 